\title{
Facilitated Diffusion
}

National Cancer Institute

\section{Source}

National Cancer Institute. Facilitated Diffusion. NCI Thesaurus. Code C82345.

Transport of substances across a biological membrane from an area of higher concentration to an area of lower concentration by means of a carrier molecule. 